\title{
Interaction between the Belousov-Zhabotinsky reaction and lipid membranes: a kinetic investigation
}

\author{
F. M. Pulselli ${ }^{1}$, M. Catalucci ${ }^{1}$, F. Rossi $^{1,2}$ \& N. Marchettini ${ }^{1}$ \\ ${ }^{1}$ Department of Chemistry, Siena University, Italy \\ ${ }^{2}$ Department of Chemistry, Brandeis University, USA
}

\begin{abstract}
Far-from-equilibrium oscillating chemical reactions are among the simplest systems showing complex behaviours and emergent properties, when coupled with diffusion they can behave as excitable media and support impulse and wave propagation. The reaction-diffusion approach has been widely employed to explain patterns generation in a great array of biological processes, like limb development and skin pattering.

During the past years our group has been studying the mechanisms of pattern formation and wave propagation occurring when the well known BelousovZhabotinsky (BZ) reaction is carried out in membranes, i.e. the lamellar phases generated by the. 1,2-dipalmitoyl-sn-glycero-3-phosphocoline (DPPC).

Many kinetic parameters of the BZ reaction were found to be influenced by the addition of increasing amounts of DPPC. The aggregates in solution are likely capable of interacting with many of the BZ intermediates, like radicals and molecular bromine. In this paper we will illustrate the results coming from a kinetic study on the temporal and spatio-temporal modifications (increase in amplitude of oscillations, increase of the lifespan of the oscillatory regime, etc.) induced on the BZ reaction by the presence of DPPC.
\end{abstract}

Keyword: Belousov-Zhabotinsky, biomimetics membrane, chemicals oscillators, thermodynamics in nature.

\section{Introduction}

The Belousov-Zhabotinsky (BZ) reaction is the most famous and thoroughly studied chemical oscillator. Initially proposed as a simplified scheme of a 
metabolic pathway (Krebs cycle), it was subsequently adopted as a more general model for certain major nonlinear phenomena, e.g. enzymatic catalysis, biological oscillations and pattern formation. It represents the catalytic oxidation of an organic substrate with active methylenic hydrogens, generally malonic acid $\mathrm{CH}_{2}(\mathrm{COOH})_{2}$, (MA), by bromate ions in a strongly acidic aqueous solution. The general stoichiometry has the form:

$$
2 \mathrm{BrO}_{3}^{-}+3 \mathrm{MA}+2 \mathrm{H}^{+} \rightarrow 2 \mathrm{BrMA}+4 \mathrm{H}_{2} \mathrm{O}+3 \mathrm{CO}_{2}
$$

where BrMA is bromomalonic acid $\left(\mathrm{BrCH}(\mathrm{COOH})_{2}\right)$. The most used catalysts are metal redox couples, such as $\mathrm{Ce}(\mathrm{IV}) / \mathrm{Ce}(\mathrm{III})$ or ferriin/ferroin $\left(\mathrm{Fe}(\mathrm{phen})_{3}\right.$ $\left.{ }^{3+} / \mathrm{Fe}(\mathrm{phen}){ }_{3}{ }^{2+}\right)$. Complexes that show markedly different colour in different oxidation states, like ferroin, are often used to visualize patterns and structures generated by the BZ reaction when coupled with transport phenomena. The BZ reaction mechanism is very complex, and the latest proposed full models for the cerium catalyzed reaction involves more than 80 elemental steps.

In the study of chemical systems characterized by nonlinear dynamics, such as the BZ reaction, surfactants are often used as perturbing agents. Surfactants are surface acting agents which belong to the wide class of the amphiphilic compounds, and they are able to form a great variety of self-assembling structures in solution, all of which share the characteristic of compartmentalization between polar and non polar regions, with defined boundary interfaces. Surfactant molecules generally consist of a hydrophilic head of various nature (ionic or non-ionic) and a hydrophobic tail which is usually linear or branched, with or without unsaturated bonds. This dual nature is the origin of the capability of the molecule of self-assembling in aqueous solvents in thermodynamically stable aggregates called "micelles". The surfactant concentration at which the reversible association of a certain number of monomers leads to aggregates (micelles) is the "critical micellar concentration" $(\mathrm{cmc})$. A micelle is therefore characterized by three solubilization sites [6,7]. They are the hydrophobic core, the palisade layer, with the polar portion in contact with the micellar surface and the hydrophobic portion directed toward the core, and the hydrophilic shell.

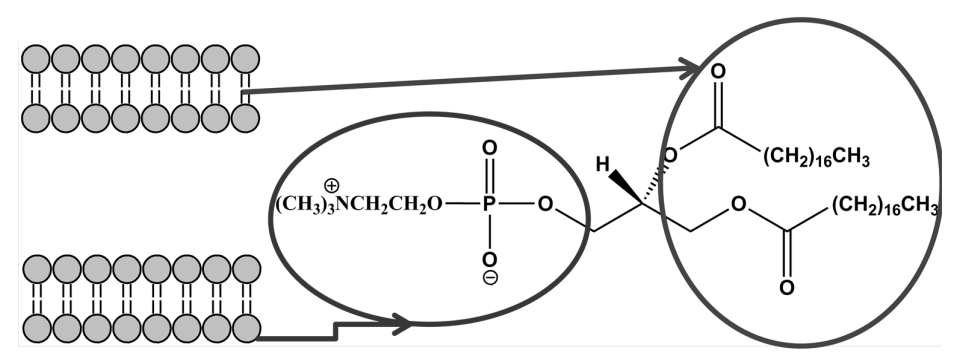

Figure 1: Structure of the bilayers (on the left) generated by the selfassembly of the DPPC molecules (on the right). The BZ reaction is confined in the water compartment between the polar heads of the lamellar phase. 
When a reaction takes place in the presence of surfactant aggregates, the chemical reactivity is obviously dependent on the solubilisation site of the reactants.

Among the other surfactants, phospholipids have attracted increasing interest as biomimetic matrices for their close resemblance to biological membranes [8, 9]. The most commonly chosen lipids to build up model membranes consist of a glycerol (propan-1,2,3-triol) backbone linked to two fatty acid chains, to a phosphate group and to a choline end. In particular, in our experiments we used a saturated amphiphile with 16 carbon hydrophobic tails, the 1,2-dipalmitoyl-snglycero-3-phosphocoline (DPPC), which is able to self-assembly in a lamellar structure (Figure 1). In the past we found that $\mathrm{BZ}$ reaction is able to generate an interesting array of moving and stationary patterns, when performed in the water phase of a DPPC lamellar stack $[4,5]$. The structural changes induced by the BZ reaction to the lipid bilayers were investigated by means of NMR [10] and Small Angle X-Ray Scattering [11] techniques. We are now undertaking a systematic study to understand the modifications of the BZ oscillating mechanism induced by the lipids. In this paper we will illustrate the results coming from a kinetic analysis of the main BZ oscillating parameters.

\section{Experimental}

All experiments were performed in a $20 \mathrm{ml}$ beaker with constant magnetic stirring. The reaction was followed by monitoring the redox potential of the solution as a function of time with a platinum microelectrode (Amel mod. 805/SPG/4) coupled with a saturated calomel reference electrode (Amel mod. 383/SHG/6). The potential was recorded with a personal computer connected to a voltmeter Amel model 338. Both the BZ reagents (SIGMA) and the DPPC (Lipoid) were used as received.

A total solution volume of $15 \mathrm{~mL}$ and the following initial composition were used for all the experimental runs:

- Malonic acid $0.30 \mathrm{~mol} \mathrm{dm}^{-3}$

- $\mathrm{NaBrO}_{3} 0.06 \mathrm{~mol} \mathrm{dm}^{-3}$

- $\mathrm{H}_{2} \mathrm{SO}_{4} 0.38 \mathrm{~mol} \mathrm{dm}^{-3}$

- Ferroin $5 \times 10^{-4} \mathrm{~mol} \mathrm{dm}{ }^{-3}$

The recorded time-series were analyzed by means of the software OriginPro 8.0. Table 1 and Figure 2 report the oscillating parameters taken into account in the present study. All parameters are averaged over the first 5 oscillatory cycles for each experiment.

\section{Results}

All of the BZ oscillatory parameters are significantly influenced when DPPC is added to the solution. The most evident changes are induced in the reaction lifespan, as can be seen in the example of Figure 3, where the last hours of two reactions are reported. It is also evident that the oscillatory regime is 
Table 1: $\quad$ Oscillatory parameters with their units.

\begin{tabular}{|c|c|}
\hline$<\mathrm{T}>/ \mathrm{s}$ & Average oscillatory period (s): \\
\hline$<\mathrm{T}_{\text {autocat }}>/ \mathrm{s}$ & $\begin{array}{c}\text { Average period included between a } \\
\text { minimum and the following } \\
\text { maximum }\end{array}$ \\
\hline$<\mathrm{T}_{\text {inhibit }}>/ \mathrm{s}$ & $\begin{array}{c}\text { Average period included between a } \\
\text { maximum and the following } \\
\text { minimum }\end{array}$ \\
\hline
\end{tabular}

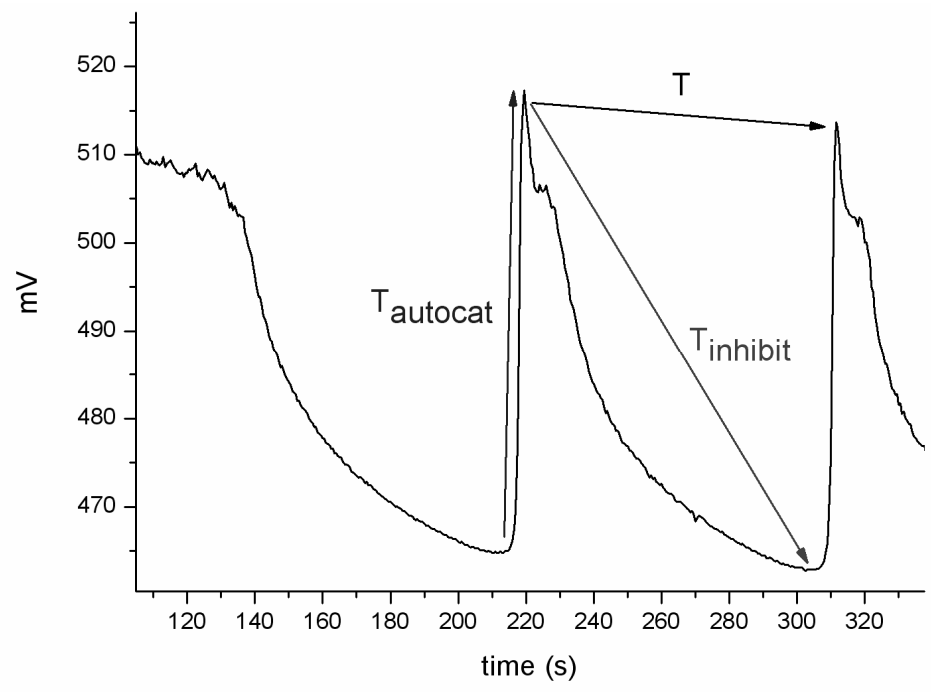

Figure 2: $\quad$ Measured oscillatory parameters.

qualitatively different in the sample containing DPPC (Fig.3a)) compared to the water system (Fig. 3b)). The BZ system involves a large number of intermediates with different characteristics (hydrophobic/hydrophilic, neutral/charge, etc.) and the DPPC may likely induce considerable effects on many aspects of the reaction. The polar heads of DPPC form a charged surface, in contact with the water phase, able to interact both with the ions and the solvent. Moreover, the hydrophobic moieties inside the bilayers can trap non-polar species, such as $\mathrm{Br}_{2}$ which is a key intermediate (inhibitor) in many steps of the BZ reaction. Other effects of the presence of lipid aggregates may involve changes in some physical properties of the reaction mixture, such as surface tension, viscosity and ionic strength. At this stage it is difficult to understand how the lipid interacts with the overall chemistry of the BZ reaction and how it influences the total number of the oscillations and their dynamical regime. 

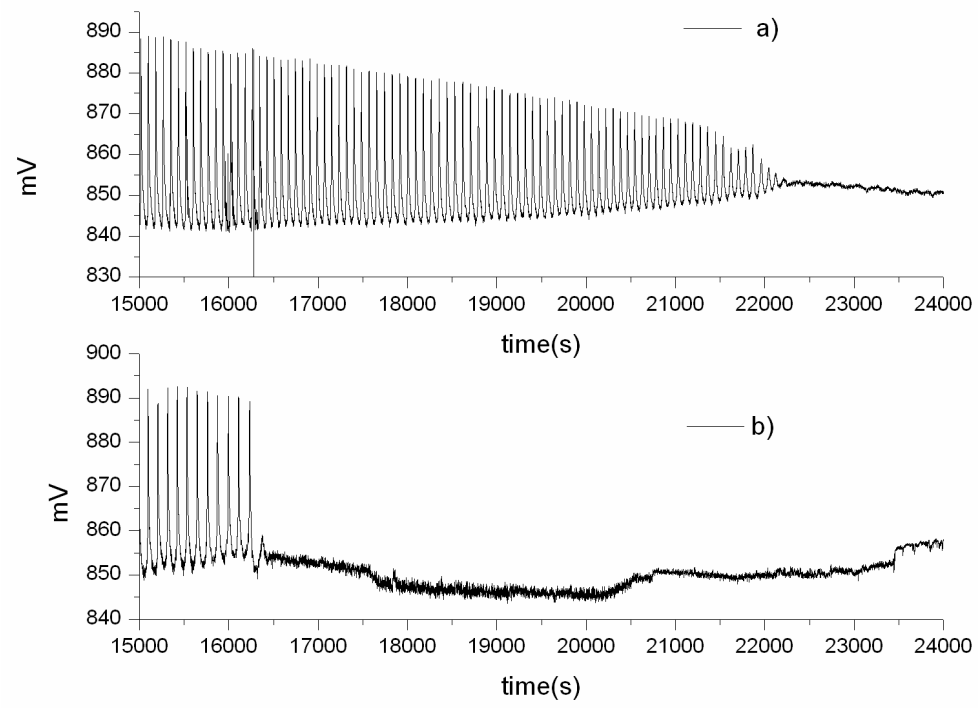

Figure 3: Last hours of the oscillatory regime of two BZ reactions in a) DPPC $3 \% \mathrm{w} / \mathrm{w}$ and b) water system.

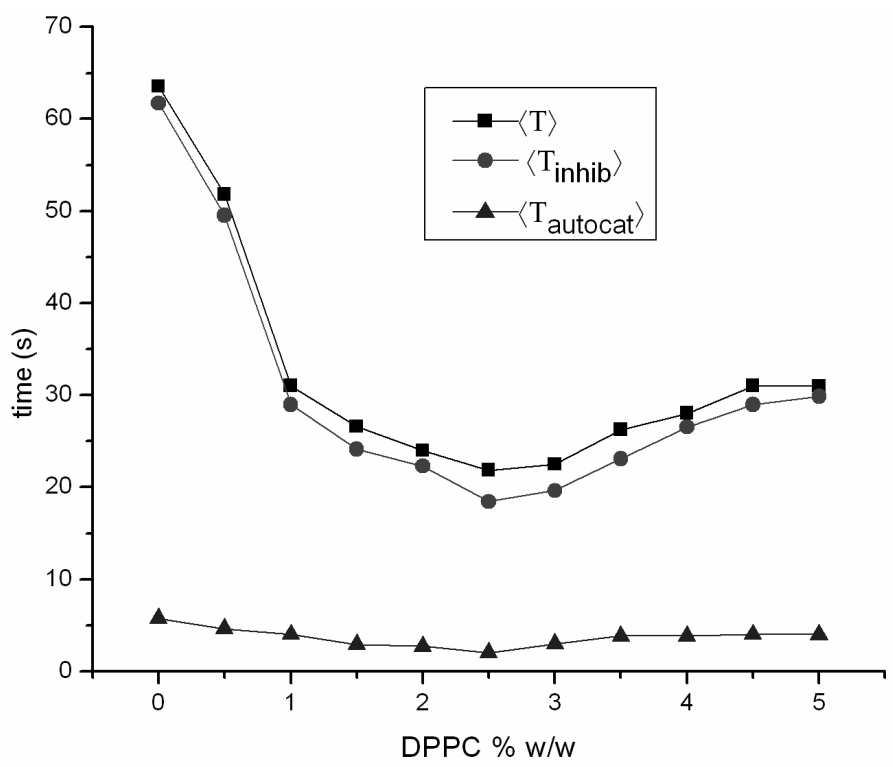

Figure 4: Metric oscillations. 

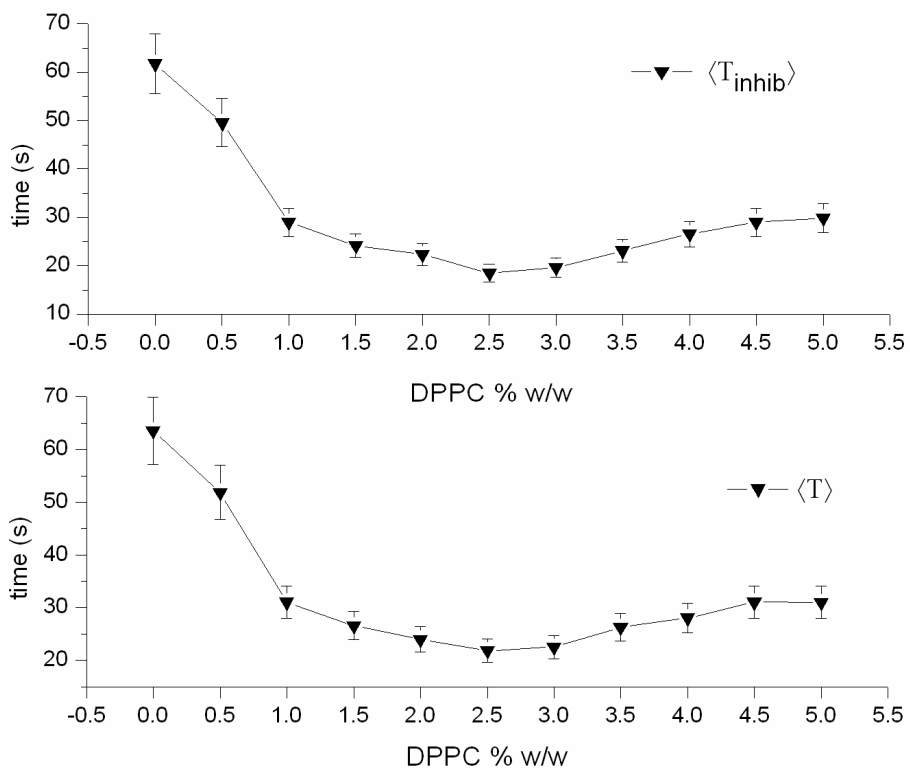

Figure 5: Average period and average period of inhibition.

Nevertheless, we can focus our attention on other parameters which are regulated by relatively simpler mechanisms. A nonlinear response of $\langle\mathrm{T}>$ to the increasing concentration of DPPC was found. In fact, after an initial decrease, the average oscillation period starts to grow when [DPPC] $>2.5 \%$, even if it never reaches the initial value (Figures 4 and 5). A similar behavior has been found in the past for other surfactants $[12,13]$.

Any oscillation cycle is composed by a part in which the concentration of the oxidized form of the catalyst (ferriin) increases (autocatalytic process) and by a second one in which it is consumed (inhibitory process). One of the processes responsible for the ferriin consumption is its reaction with the bromomalonic acid during the reaction $[11,13]$

$$
2 \mathrm{Fe}(\text { phen })_{3}{ }^{3+}+2 \mathrm{BrMA} \rightarrow f \mathrm{Br}^{-}+2 \mathrm{Fe}(\text { phen })_{3}{ }^{2+}+\text { other products }
$$

According to Edelson [14], reaction (2) is the most important step in determining the length of $<\mathrm{T}>$. In fact, in Figure 4 we see that, if we split $<\mathrm{T}>$ in $\left\langle\mathrm{T}_{\text {autocat }}\right\rangle$ and $\left\langle\mathrm{T}_{\text {inhibit }}\right\rangle$, the contribution of the latter to the total length of $\langle\mathrm{T}\rangle$ is predominant. The initial decrease of the oscillation period, it is possibly due to the role of the lipid as a radical scavenger. The same behavior was observed for the zwitterionic surfactant CHAPS 3-[(3-cholamidopropyl)dimethylammonio]-1 propanesulfonate] [13].

It is interesting to note from Figures $4-6$, that both $\left\langle\mathrm{T}_{\text {autocat }}\right\rangle$ and $\left\langle\mathrm{T}_{\text {inhibit }}\right\rangle$ have the same threshold behavior at $[\mathrm{DPPC}]=2.5 \%$. Since the length of the autocatalytic and the inhibitory parts are regulated by different chemical 


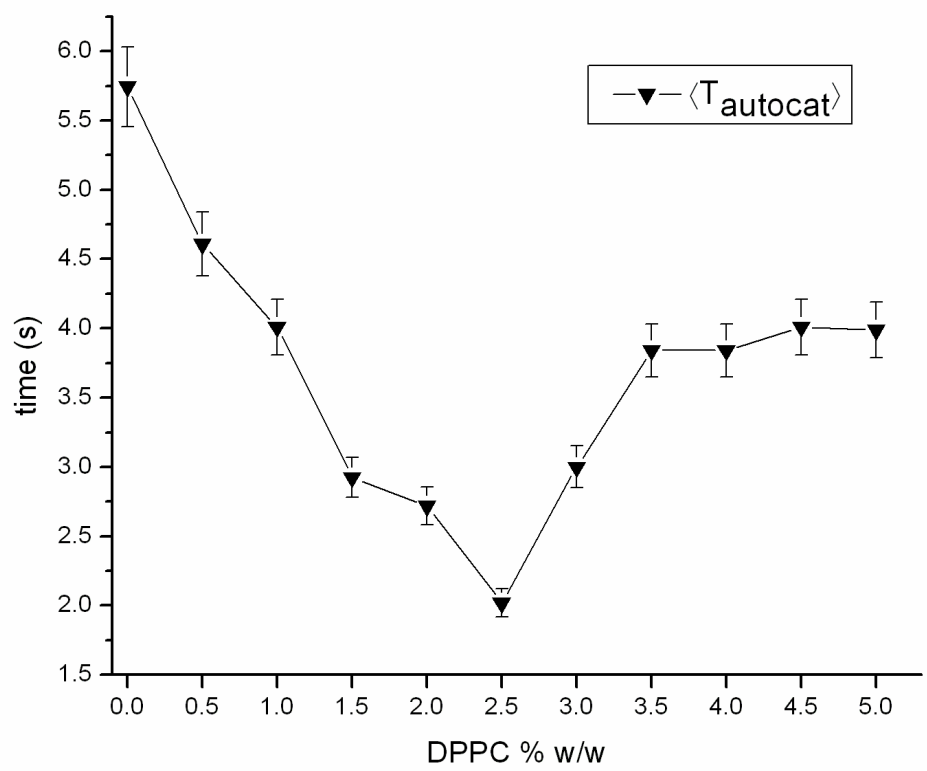

Figure 6: Average period of autocatalysis.

processes, it is hard to attribute these trends to the same chemical interaction mechanism. Most probably, by increasing the lipid concentration the aggregates structure undergoes to a physical transition (e.g. vesicles to bilayers). In this case, several physical properties (viscosity, ionic strength, surface tension, etc.) can be affected and their effect can be fundamental in determining the oscillatory behavior. Further studies in this direction are already planned.

\section{Conclusions}

The oscillating Belousov-Zhabotinsky reaction has been shown to respond in a peculiar way to addition of increasing amounts of DPPC in batch reactors under stirring. It can be concluded that DPPC acts as a catalyst for the reaction, increasing the average oscillation frequency, though with a nonlinear trend. At this stage it is very difficult to understand the interaction mechanism between the BZ intermediates and the lipids, nevertheless our kinetic measurements provide an interesting starting point to be extended with further studies.

\section{Acknowledgements}

This research was supported by a Marie Curie International Outgoing Fellowship (crossdiffusionrossi - 237849) within the $7^{\text {th }}$ European Community Framework Programme. Thanks are due to Dr. Sandra Ristori for helpful discussions. 


\section{References}

[1] E. Tiezzi, Steps Towards an Evolutionary Physics, Wit Press, Southampton (UK), pp. 157, 2006.

[2] V.K. Vanag and I.R. Epstein, "Pattern formation mechanisms in reactiondiffusion systems," International Journal of Developmental Biology, vol. 53, 2009, pp. 673-681.

[3] J.D. Murray, Mathematical Biology, Berlin: Springer-Verlag, 2002.

[4] A. Magnani, N. Marchettini, S. Ristori, C. Rossi, F. Rossi, M. Rustici, O. Spalla, and E. Tiezzi, "Chemical waves and pattern formation in the 1,2dipalmitoyl-sn-glycero-3-phosphocholine/water lamellar system," Journal of the American Chemical Society, vol. 126, 2004, pp. 11406-11407.

[5] F. Rossi, S. Ristori, M. Rustici, N. Marchettini, and E. Tiezzi, "Dynamics of pattern formation in biomimetic systems," Journal of Theoretical Biology, vol. 255, 2008, pp. 404-412.

[6] J.H. Fendler, Membrane Mimetic Chemistry, New York: John Wiley \& Sons Inc, 1983.

[7] J.H. Fendler, "Interactions and Kinetics in Membrane Mimetic Systems," Annual Review of Physical Chemistry, vol. 35, 1984, pp. 135-157.

[8] J.K. Parrish and L. Edelstein-Keshet, "Complexity, Pattern, and Evolutionary Trade-Offs in Animal Aggregation," Science, vol. 284, Apr. 1999, pp. 99-101.

[9] P. Walde, Prebiotic Chemistry: From Simple Amphiphiles to Protocell Models, Berlin: Springer-Verlag, 2005.

[10] N. Marchettini, S. Ristori, F. Rossi, and M. Rustici, "An experimental model for mimicking biological systems the Belousov-Zhabotinsky reaction in lipid membranes," International Journal of Ecodynamics, vol. 1, 2006, pp. 55-63.

[11] S. Ristori, F. Rossi, G. Biosa, N. Marchettini, M. Rustici, and E. Tiezzi, "Interplay between the Belousov-Zhabotinsky reaction-diffusion system and biomimetic matrices," Chemical Physics Letters, vol. 436, 2007, pp. 175-178.

[12] F. Rossi, R. Lombardo, L. Sciascia, C. Sbriziolo, and M.L.T. Liveri, "Spatio-Temporal Perturbation of the Dynamics of the Ferroin Catalyzed Belousov-Zhabotinsky Reaction in a Batch Reactor Caused by Sodium Dodecyl Sulfate Micelles.," Journal of Physical Chemistry B, vol. 112, 2008, pp. 7244-7250.

[13] A. Paul, "Observations of the Effect of Anionic, Cationic, Neutral, and Zwitterionic Surfactants on the Belousov-Zhabotinsky Reaction," Journal of Physical Chemistry B, vol. 109, 2005, pp. 9639-9644.

[14] D. Edelson and V.M. Thomas, "Sensitivity analysis of oscillating reactions. 1. The period of the Oregonator," Journal of Physical Chemistry, vol. 85, 1981, pp. 1555-1558. 